\title{
Is There a Need to Drain Cervical Cysts in Women with Lower Genital Tract Symptoms?
}

\author{
Atef Darwish MD PhD ${ }^{1}$, Alaa M. Ismail MD. ${ }^{1}$, Michael N. Agban PhD. ${ }^{2}$ and Hassan S \\ Kamel MD ${ }^{1}$ \\ ${ }^{1}$ Department of Obstetrics and Gynecology, Woman's health University center, Assiut University, Assiut, Egypt. \\ ${ }^{2}$ Department of Microbiology and Immunology. Faculty of Medicine, Assiut University, Assiut, Egypt.
}

\begin{abstract}
:
Objectives: To determine whether gynecologists should treat cervical cysts discovered on speculum examination or by ultrasonography.

Materials and methods: A prospective Cohort study done at the Woman's Health Center of Assiut University, Egypt as a tertiary care referral facility. It comprised cases with clinical and sonographic diagnosis of cervical cysts. Cervical swabs as well as cysts' aspirates were examined bacteriologically and cytologically. Cyst aspirate of these patients were evaluated for IL8, IL10 and MMP-9. The main outcome measures included bacteriologic, cytologic and immunochemical findings of cervical cysts aspirate.

Results: Cervical cysts associated with negative cervical swabs were diagnosed in 75 cases. The mean size of the cervical cyst was $2.18 \pm 0.609 \mathrm{~cm}$. It was found that $61.4 \%$ of cysts were cytologically and bacteriologically infected despite negative cervical swabs. Chlamydia Trachomatis, Gardenella Vaginalis, GBS and Mycoplasma hominis were detected in $13.33 \%, 24 \%, 14.67 \%$ and $14.67 \%$ of infected cysts respectively. Aspirate of the infected cysts had elevated IL-8 $(\mathrm{P}=0.000)$, and had increased IL-10 $(\mathrm{P}=0.000)$ in comparison with non infected cysts, while increased MMP-9 was higher in patients with persistent vaginal discharge and patients with deep dyspareunia more than the patients with unexplained infertility $(\mathrm{P}=0.000)$.

Conclusions: Cervical cysts should not be ignored as a potential cause of common gynecological symptoms especially large sized cysts. Being infected and associated with elevated inflammatory markers in $61.4 \%$ in this study would suggest routine prompt management of cervical cysts whenever clinically or sonographically diagnosed.
\end{abstract}

Keywords: Cervical Cysts, Hysteroscopy, Transvaginal Ultrasonography, Bacteriology, Cytology.

\section{INTRODUCTION}

For a long time, it is believed that tiny cervical cysts represent a normal change of the transformation zone of the cervix during the process of metaplasia of the columnar epithelium. Normal colposcopic appearance of the cervix may include cervical cysts, commonly called Nabothian retention cysts, with exaggerated but normal overlying blood vessels as seen by saline technique with green filter during colposcopy (1). Research on cervical cysts in literature is very scanty. Cervical cysts have received little attention both from the standpoint of characterization (either histologically or microbiologically) and their connection with clinical problems. These commonly seen cysts are usually discovered during routine vaginal speculum examination or during transvaginal ultrasonography (2). Clinically, cervical cysts most often look like tiny papules and are filled with mucous (3). These cysts may be single or multiple between 2 millimeters and 10 millimeters in diameter (4). Cysts usually bulge towards ectocervix, where they appear as smooth and clear swellings up to the size of a pea and usually firmer the surrounding cervix (5). Nabothian cysts rarely cause any problems and need no treatment (6). However, if they are associated with chronic cervicitis (which is likely to cause blockage of ducts), there is a general consensus among gynecologists to open them during cervical cauterization. Whether they represent a nidus for infection or not remains to be proved (7). Inflammatory markers can indicate the occurrence of cervical inflammation (8) but may not indicate infection of these retention cysts. The impact of these cysts on fertility as well as the patient's common gynecological complaint and health related quality of life is still unknown (7). Proposed mechanisms of impaired fertility secondary to

${ }^{1}$ Corresponding Author: atef_darwish@yahoo.com 
cervical cysts would include mechanical compression of the endocervical canal, or chronic irritation and infection with increased level of inflammatory cytokine (8). Interleukin-8 (IL-8) is a chemokine produced by macrophages and other cell types such as epithelial cells. It functions as a chemo attractant, and is also a potent angiogenic factor. Interleukin-8 is often associated with inflammation (9). Interleukin - 10 (IL-10), also known as human cytokine synthesis inhibitory factor (CSIF), is an anti-inflammatory cytokine. In humans IL-10 is encoded by the IL10 gene. This cytokine is produced primarily by monocytes and to a lesser extent by lymphocytes. This cytokine has pleiotropic effects in immunoregulation and inflammation (8). Matrix metalloproteinases (MMPs) form a family of enzymes with major actions in the remodeling of extracellular matrix components. MMP-9, also called Gelatinase $\mathrm{B}$, is the most complex family member in terms of domain structure and regulation of its activity. The main function of MMP-9 is degradation of the extracellular physiologic function. MMP-9 also contributes to pathological conditions (9).

At our institution, there is an increasing interest in the uterine cyst diagnosis and management. We recently published a protocol of management of symptomatic uterine cysts (10). In the previous study, it was clinically noticed that the patient's quality of life improved after proper minimally invasive management of uterine cysts. This study aims to determine whether gynecologists should treat cervical cysts discovered on speculum examination or by ultrasonography.

\section{PAtients AND Methods}

This study was conducted at Woman's Health University Center, Assiut University, Assiut, Egypt between March 2009 and April 2014 and comprised symptomatic patients attending the gynecologic outpatient clinic with clinical or sonographic diagnosis of cervical cysts. They were in child bearing period (18-44 ys). A written informed consent was taken. Patients had the right to refuse to participate and/or withdraw from the study at any time without being denied their regular full clinical care. Personal information as well as data collected was subjected to confidentiality. The Institutional Review Board (IRB) of Assiut faculty of medicine approved the study. Exclusion criteria included symptomatizing women with clinical or sonographic diagnosis of a possible cause of infection like pelvic inflammatory disease (PID), adnexal lesion, endometriosis or uterine lesion. Positive cervical swab for infection was an exclusion from this study. Included patients with negative cervical swabs were 75 cases. Forty four cases were easily diagnosed by speculum examination while 31 cases were referred to the outpatient clinic after accidental TVS diagnosis of cervical cysts again in symptomatic patients. Among the clinically diagnosed cervical cysts, TVS was requested for 24 cases whenever big cysts more than $1 \mathrm{~cm}$ were clinically seen to exclude other intracervical or high cysts. Office hysteroscopy was requested in only 8 cases whenever TVS diagnosed cervical cysts not seen on speculum examination. All cervical cysts were aspirated using a 14 gouge aspiration needle with high manual suction power as the cystic fluid was viscid in all cases. If the cysts were inaccessible clinically, sonographically guided or hysteroscopically guided aspiration were performed as previously described by our team (10). Before cyst aspiration, the cervix was meticulously washed with sterile saline and any cervical secretions were repeatedly removed. Cytologic examination of a drop of aspirate was made to test for some inflammatory markers like the presence of PMN or others and a smear was stained by Papanicolau stain to exclude malignancy. For bacteriologic assessment, the aspirated fluid were immediately transferred to Amies transport medium (Himedia), pleuropneumonia-like organism broth (PPLO) (Himedia - Cat. No. M266), and brain heart infusion (BHI) (Himedia - Cat. No. M210), to isolate Mycoplasma hominis. The plates were kept under microaerophilic conditions at $37^{\circ} \mathrm{C}$. Liquid media were examined daily for 10 days for the color change indicating growth. Other media like columbia agar base (Himedia - Cat. No. M144) and MacConkey agar (Himedia - Cat. No. MM081) was used to identify other organisms by conventional methods. Vaginalis agar medium was used to detect Gardnerella vaginalis (Himedia Cat. No. M1057). Part of aspirated fluid were fixed in $10 \%$ formalin, paraffin-embedded, and stained with hematoxylin-eosin stain for microscopic study. Part of the fluid were fixed on slides, frozen in acetone, and subjected to a direct immunofluorescence assay (IFA) with fluorescence isothiocyanate conjugated anti-chlamydia trachomatis monoclonal antibodies (ImagenTM Chlamydia, Dako Cytomation, UK). Detection of group B streptococci (GBS) by specific antiserum on the isolated colonies (HiStrep - Latex test kit - Himedia LK06-50NO) was tested in all cases. The isolated organisms were confirmed biochemically using API system (20A Biomerrieux RES 20300). Antibiogram for isolated microorganisms to detect the proper antibiotic was done against Penicillin, cephalosporins, macrolides, and quinolones (Himedia), Patients with proved infected cysts received full course of antibiotic according to its antibiogram.

For immunological assay of inflammatory cytokines, cyst aspirate of these patients were assayed for human MMP9 (ELISA, Bender MedSystems GmbH, Austria) the plate was coated by monoclonal Ab to MMP-9. IL-8 (ELISA, Immunotech IM2237-8) the plate was coated by monoclonal Ab to IL-8. and IL-10 (ELISA, Immunotech IM1987) 
the plate was coated by monoclonal Ab to IL-10. All cases were subjected to the established management plan at our institution as previously published (10). Thereafter, all women were examined again and tested in the same way as before treatment. Data entry was done using Epi-Info 6.04 computer software package, while statistical analysis was done using SPSS version 17 (Chicago, USA) statistical software packages. Quality control was done at the stages of coding and data entry. Data were presented using descriptive statistics in the form of frequencies and percentages for qualitative variables, and ranges, means and standard deviations, medians and quartiles for quantitative variables in box plots. The comparability of baseline characteristics according to outcome was ascertained by Student's $t$ test (unpaired $t$ test) for continuous variables and Mann Whitney $U$ test when appropriate, and $\mathrm{X}^{2}$ test (Chi square test) for categorical variables. To evaluate the univariate relation between variables, Pearson's correlation coefficient was calculated. Values were considered significant if $p$ value $\leq 0.05$.

\section{RESULTS}

This study comprised 75 patients with symptomatic cervical cysts and a negative cervical swab. Abnormal excessive vaginal discharge was reported in 32 cases, 28 cases presented with pelvic pain, backache and/or dyspareunia, and 15 cases with unexplained infertility. However, more than one complaint was reported in many cases. Table (1) summarizes their demographic, clinical, sonographic and hysteroscopic data. Failed sonographic access or aspiration was encountered in 7 cases (7\%) of deep seated or small cysts. The mean size of the cervical cyst was $2.38 \pm 0.61 \mathrm{~cm}$. Solitary cyst was diagnosed in $31(41.3 \%)$ of patients and multiple cysts were seen in 44(58.7\%). As regarding the site of the cysts, 31 cases was superficial (41.3\%) and 44 cases was a deep seated cyst (58.6\%). Tables (2) summarize the comparisons between the infected and the non infected cases. Infected cysts were bacteriologically diagnosed in 46 of cases $(61.33 \%)$ while 29 cases $(38.66 \%)$ were not infected $(\mathrm{p}=0.03)$. Parity was higher in the infected group $2.50 \pm 0.15(\mathrm{p}<0.008)$. Likewise, the local inflammatory cytokines were higher in the infected cysts $(\mathrm{p}<0.000)$. As regarding the numbers and the size of the cysts, solitary cysts were common in the infected cysts $(\mathrm{p}<0.000)$ but the larger size was in the non infected cysts.1 Table (3) shows the frequency of different isolated microorganisms among patients. Chlamydia trachomatis was diagnosed in $13.33 \%$, while Gardenella vaginalis was diagnosed in $24 \%$ of cases. In $14.75 \%$ of cases the infective organism was group b Streptococcus (GBS), Mycoplasma hominis was found in $14.75 \%$ of cases. The rest $13.3 \%$ of cases were due to other forms of organisms. Cytologic evaluation of the cyst aspirate revealed no malignant cells in all cases but showed ample inflammatory cells in most cases (68 cases,90.7\%). Figure (1) shows the correlation between the size of cyst and the level of inflammatory cytokines. There was highly significant negative correlation between the size of cyst and the level of IL-8 and IL-10 but not the number or site of the cysts. The level of inflammatory cytokines (IL-8, IL-10 and MMP-9) was high in all infected cases; the levels were higher in 32(42.67\%) patients with persistent vaginal discharge and 28(37.33\%) patients with deep dyspareunia more than the 15(20\%) patients with unexplained infertility. Figure (2) shows the immunofluorescence assay of Chlamydia trachomatis organism. All women received the appropriate treatment. Biochemical reassessment revealed marked improvement of measurements suggestive of inflammation. Since we included infertile women, the clinical results were excluded to allow proper follow-up of this group.

Table1. Demographic, clinical, sonographic and hysteroscopic data

\begin{tabular}{|c|c|}
\hline Item & Descriptive \\
\hline $\begin{array}{l}\text { Age } \\
\text { Mean } \pm \text { S.D }\end{array}$ & $28.23 \pm 5.21$ \\
\hline $\begin{array}{l}\text { Parity } \\
\text { Mean } \pm \text { S.D }\end{array}$ & $2.13 \pm 1.02$ \\
\hline IL- 8 & $200.90 \pm 90.32$ \\
\hline IL-10 & $273.71 \pm 120.30$ \\
\hline mmp & $0.613 \pm 0.49$ \\
\hline Cyst size & $2.38 \pm 0.61$ \\
\hline \begin{tabular}{cc}
\multicolumn{2}{l}{ No of cysts } \\
$\qquad \quad$ Solitary \\
$\bullet \quad$ Multiple
\end{tabular} & $\begin{array}{l}31(41.3 \%) \\
44(58.7 \%)\end{array}$ \\
\hline $\begin{array}{l}\text { Complaint } \\
\text { Abnormal Vaginal discharge } \\
\text { Pelvic pain. } \\
\text { Un explained infertility }\end{array}$ & $\begin{array}{c}32(42.67 \%) \\
28(37.33 \%) \\
15(20 \%)\end{array}$ \\
\hline
\end{tabular}


American Research Journal of Nursing, Volume 1, Issue 5, 2015

ISSN 2379-2922

Table2._Infected versus non-infected cysts.

\begin{tabular}{|c|c|c|c|}
\hline Item & Noninfected"n=29" & Infected"n=46" & p.value \\
\hline Age & $27.1 \pm 4.21$ & $30.58 \pm 7.74$ & $\mathbf{P}<0.03 *$ \\
\hline Parity & $1.82 \pm 0.19$ & $2.50 \pm 0.15$ & $\mathbf{P}<0.008$ *** \\
\hline IL-8 & $36.13 \pm 6.56$ & $304.78 \pm 112.99$ & $\mathbf{p}<0.000 * *$ \\
\hline IL-10 & $30.80 \pm 9.11$ & $426.84 \pm 35.29$ & $\mathbf{p}<0.000 * *$ \\
\hline MMP9 & $4.63 \pm 2.81$ & $7.51 \pm 0.61$ & $\mathbf{p}<0.02 * *$ \\
\hline Cyst size & $2.61 \pm 0.67$ & $2.24 \pm 0.54$ & $\mathrm{p}<0.01 *$ \\
\hline $\begin{array}{l}\text { Cyst No. } \\
\text { Solitary } \\
\text { Multiple }\end{array}$ & $\begin{array}{c}9(31.0 \%) \\
20(69.0 \%)\end{array}$ & $\begin{array}{l}22(47.8 \%) \\
24(52.2 \%)\end{array}$ & $\begin{array}{l}\mathbf{p}<0.000 * * \\
\mathbf{p}=0.115 \\
\text { n.s }\end{array}$ \\
\hline Item & $\underline{\text { Noninfected"n=29" }}$ & Infected"n=46" & p.value \\
\hline$\overline{\text { Age }}$ & $27.1 \pm 4.21$ & $30.58 \pm 7.74$ & $\mathbf{P}<0.03 *$ \\
\hline Parity & $1.82 \pm 0.19$ & $2.50 \pm 0.15$ & $\mathrm{P}<0.008 * *$ \\
\hline IL-8 & $36.13 \pm 6.56$ & $304.78 \pm 112.99$ & $\mathrm{p}<0.000 * *$ \\
\hline IL-10 & $30.80 \pm 9.11$ & $426.84 \pm 35.29$ & $\mathbf{p}<0.000 * *$ \\
\hline MMP9 & $4.63 \pm 2.81$ & $7.51 \pm 0.61$ & $\mathbf{p}<0.02 * *$ \\
\hline Cyst size & $2.61 \pm 0.67$ & $2.24 \pm 0.54$ & $\mathrm{p}<0.01 *$ \\
\hline $\begin{array}{l}\text { Cyst No. } \\
\text { Solitary } \\
\text { Multiple }\end{array}$ & $\begin{array}{c}9(31.0 \%) \\
20(69.0 \%)\end{array}$ & $\begin{array}{l}22(47.8 \%) \\
24(52.2 \%)\end{array}$ & $\begin{array}{l}\mathbf{p}<0.000 * * \\
\mathbf{p}=0.115 \\
\text { n.s }\end{array}$ \\
\hline
\end{tabular}

Table3. Main microorganisms detected in all cases.

\begin{tabular}{|c||c||}
\hline \hline Item & NO (\%) \\
\hline \hline $\begin{array}{c}\text { Microorganism : } \\
\text { Chlamydia Trachoma }\end{array}$ & $10(13.33 \%)$ \\
\hline \hline Gardenella vaginalis & $18(24 \%)$ \\
\hline \hline GBS & $11(14.67 \%)$ \\
\hline \hline Mycoplasma Hominis & $11(14.67 \%)$ \\
\hline Others & $10(13.33 \%)$ \\
\hline
\end{tabular}

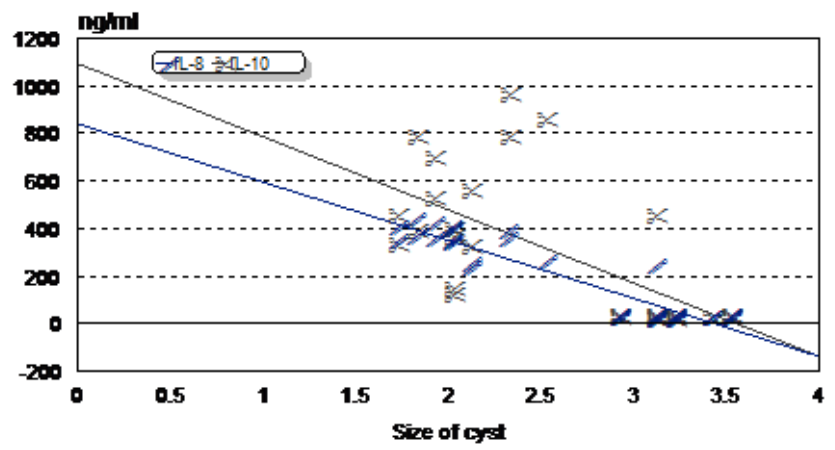

Fig1. Correlation between the size of cyst $(\mathrm{cm})$ and the level of inflammatory cytokines $(\mathrm{ng} / \mathrm{ml})$.

Fig (1): shows the correlation between the size of cyst and the level of inflammatory cytokines. There is high significant negative correlation between the size of cyst and the level of IL-8 and IL-10 but not the number or site of the cysts. 
American Research Journal of Nursing, Volume 1, Issue 5, 2015

ISSN 2379-2922

Fig (2) shows the immunofluorescence assay of Chlamydia trachomatis organism.

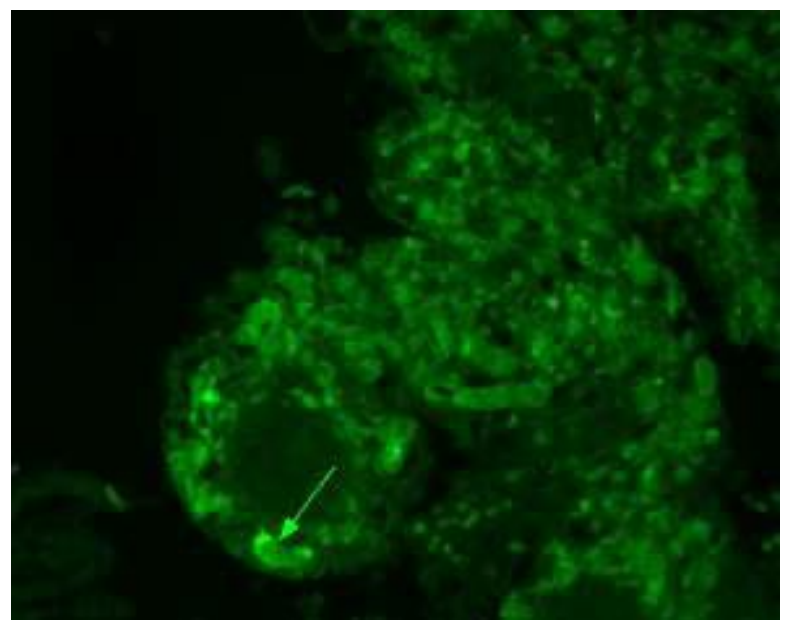

Fig2. Immunofluorescence assay of Chlamydia trachomatis organism. Note the intracytoplasmic inclusions (arrow) that is characteristic of Chlamydia trachomatis species.

\section{DISCUSSION}

The most frequently seen uterine cysts are cervical cysts which are unfortunately poorly studied in literature. Few case reports on rare types of uterine cysts were published (11, 13-15). Infected cysts account for reasonable ratio which may necessitate aspiration followed by culture and sensitivity test to the aspirate of deep, multiple or large cysts (more than $2 \mathrm{~cm}$ ), which are commonly associated positively with the patient complaint (12). It is fairly certain that most colonizing bacteria of the female genital tract are adherent to the surface epithelium and passing a needle through contaminated tissue may carry bacteria to the site of the cystic lumen. Thus, a decision to include only cases with negative cervical swab cultures was made. Moreover, we tried to assess the effect of cervical cysts on common female genital complaints, and the effect of inflamed cysts on patient's complaint. All cases of cervical cysts were easily diagnosed utilizing conventional high resolution 2D ultrasonography with clear demarcation of the sit, size and the number of cysts was done easily.

In this study, just cyst aspiration was performed since these rare cysts are usually benign in nature. Moreover, cytologic examination of the aspirated cases in this study as well as our previous study (10) revealed no evidence of malignant cells. Nevertheless, cyst aspiration is not an optimal approach. Ideally, the cyst wall should be pealed off to ensure non-reformation of the cyst. In this study, office hysteroscopy was done to evaluate the relation and the effect of the cyst to the cervical canal. Large cysts more than $2 \mathrm{~cm}$ commonly causing indentation of the cervical canal, but sometimes it protrude through the canal where it is easily to be aspirated. Small cysts $(0.5-1.5 \mathrm{~cm})$ less affecting the cervical canal but sometimes gave polypoidal protrusions. Previously, a monopolar needle for blind puncture of these cervical cysts was tested by our team (10). However, this procedure may be complicated by iatrogenic cervical atresia.

In this study, infected cysts were detected in $61.4 \%$ of aspirated cysts. On the other hand, most papers didn't try to identify the nature of the cysts (7). Chlamydia Trachomatis was detected in $13.33 \%$ of infected cysts, while Gardenella Vaginalis was detected in $24 \%$, GBS in $14.67 \%$ and Mycoplasma hominis in 14.67\%. The role of such organisms in female genital complaints is well established in medical practice. Increased inflammatory cytokines (IL-8, IL-10 and MMP-9) were detected in the infected group than the non infected one with highly significant difference for IL-8 and IL-10 in all cases, while increased MMP-9 was higher in patients with persistent vaginal discharge and patients with deep dyspareunia more than the patients with unexplained infertility. $(\mathrm{P}=0.000)$. This may explain the clinical presentations, and increased levels of inflammatory cytokines in both infected and non infected cysts especially the large cysts also may explain the obstetric career (three cases of the studied cases have recurrent second trimester abortion, and five cases with recurrent pre-labor rupture of membrane), this is in agree with other papers $(2,8,9)$ that detected high level of inflammatory cytokines in similar cases. In general, there is no normal value for levels of cytokines and all types of kits not provide levels of normal value, it's considered in comparison to normal controls. Although we didn't involve any normal cases, there is an evident difference between the levels in both infected and non infected groups which indicates the effect of such infection on level of cytokines. 
In this study, we found high significant negative correlation between the size of cyst and the level of IL-8 and IL-10, but not the MMP-9. Such relation couldn't be found between the site, or number of cysts and the level of cytokines. This may explain the relation between the size of cyst and the presence of complaint that increase by infection.

The limitations of the study include failure to obtain cyst wall for histopathologic examination to determine the cyst nature, and failed aspiration in some cases due to very small size or deep sited cysts. Further similar studies with sufficient sample size are needed to evaluate the impact of the cysts on gynecologic and obstetric complaints and the clinical effect of cyst aspiration in each group. Lastly, small sample size of this study is a real limitation. From this preliminary study, we conclude that cervical cysts should not be ignored as a potential cause of common gynecological symptoms especially large sized cysts. Being infected and associated with elevated inflammatory markers in $61.4 \%$ in this study would suggest prompt management of cervical cysts clinically or sonographically diagnosed. Further large-sample sized studies are required to define whether cyst aspiration could be a reliable line of therapy.

Disclosure: the authors have no closure of interest.

\section{REFERENCES}

[1] Kuramoto H, Sugimoto N, Iida M. Screening for cancer of the cervix with simultaneous Pap smear and colposcopy. The efficacy of pap smear and colposcopy. Eur J Gynaecol Oncol. 2011;32(1):73-6.

[2] Fridman R, Toth M, Chvyrkova I, Meroueh SO, and Mobashery S. Cell surface association of matrix metalloproteinase - 9 (gelatinase-B). Cancer Metastasis Rev. 2003; 22 (2-3): 153-166.

[3] Quaas A, and Dokras A. Diagnosis and Treatment of Unexplained Infertility. Rev Obstet Gynecol 2008; 1(2): 69-76.

[4] Mitsumori A, Morimoto M, Matsubara S, Yamamoto M, Akamatsu N, and Hiraki Y. MR appearance of adenomatoid tumor of the uterus. J Comput Assist Tomogr 2000; 24:610 -613.

[5] Kaya saracl, MD, Ramazan Kutlul, MD, Ozcan Balat et al. Evaluation of nabothian cysts with trans vaginal sonography, Journal of turgur ozal Medical Center1997; 4(2):222-224.

[6] Gleicher N, Barad D. Unexplained infertility: Does it really exist? Human Reproduction 2006; 21(8):1951-1955.

[7] Aetena Intelhealth. Nabothian (inclusion) cysts; Article reviewed by the Harvard Medical School consumer health information board 2002.

[8] Bacon K, Baggiolini M, Broxmeyer H, Horuk R, Lindley I, Mantovani A, Maysushima K, Murphy P, Nomiyama H, Oppenheim J, Rot A, Schall T, Tsang M, Thorpe R, Van Damme J, Wadhwa M, Yoshie O, Zlotnik A, Zoon K. "Chemokine/chemokine receptor nomenclature". J. Interferon Cytokine Res. 2002; 22 (10): 1067-8.

[9] Pestka S, Krause CD, Sarkar D, Walter MR, Shi Y, Fisher PB. "Interleukin-10 and related cytokines and receptors". Annu. Rev. Immunol.2004; 22: 929-79.

[10] Atef M. Darwish,, Kamal M. Zahra, Mohammad A. Bedaiwi, Mahmoud E Zakhera. Minimally invasive surgical management of symptomatic uterine cysts. Gynecological Surgery 2011; 8, 1, 19.

[11] Sang Hwa Shim·Han-Seong Kim Mee Joo·Sun Hee Chang Ji Eun KwakFlorid Cystic Endosalpingiosis of the Uterus- A Case Report. The Korean Journal of Pathology 2008; 42: 189-91.

[12] Erguvan1 R, Meydanli MM, Alkan A, Gokce MN, and Kafkasli A. Abscess in adenomyosis mimicking a malignancy in a 54-year-old woman Infect Dis Obstet Gynecol 2003;11:59-64.

[13] Ho M L., Raptis C., Hulett R., McAlister W.H., Moran K., Bhalla S. Adenomyotic cyst of the uterus in an adolescent. Pediatric Radiology 2008; 38, 11,1239-1242

[14] Langley GF. Primary echinococcal cyst of the uterus British Journal of Surgery Jan1943;30,119,278-280 Online: Dec 6 2005.

[15] Protopapas A, Milingos S, Markaki S, Loutradis D, Haidopoulos D, Sotiropoulou M, Antsaklis A. Cystic Uterine Tumors Gynecol Obstet Invest 2008; 65:275-280. 